\title{
Entre la racionalidad instrumental y el «imaginario managerial». Estrategias didácticas en la enseñanza del Management
}

\author{
Between the Instrumental Rationality and the "Managerial \\ Imaginery». Teaching Strategies in Management Classes
}

Instituto de Desarrollo Económico y Social - Centro de Antropología Social (Argentina)

Artículo recibido: 31 marzo 2016

Solicitud de revisión: 20 junio 2016

Artículo aceptado: 25 octubre 2016

Resumen

A partir del registro etnográfico correspondiente a un curso de Liderazgo en una escuela de negocios del Gran Buenos Aires, este trabajo aborda la relación entre los objetivos educacionales (el "para qué» de la enseñanza), el contenido (el «qué» de la enseñanza) y las acciones e interacciones dentro del aula. Intenta mostrar la tensión que se establece entre la lógica de la eficiencia - que estructura la tarea del sistema educativo- y la lógica vincular de un «imaginario managerial» (Alonso y Fernández Rodríguez, 2006) que tácitamente condiciona la actividad de docentes y cursantes.

Palabras clave: enseñanza, aprendizaje, crítica, imaginario managerial, management, liderazgo.

\begin{abstract}
From the ethnographic record of a Leadership course in a Business School close to Buenos Aires, this article analyzes the relationship among educational goals (purposes of teaching), content (topics to be taught) and interactions in the classroom. It attempts to underscore the tension between the instrumental rationality grounded in the educational system and the relational rationality underlying a «managerial imaginery» (Alonso $y$ Fernández Rodríguez, 2006) which tacitly conditions teaching and learning activities.
\end{abstract}

Keywords:Teaching, Learning, Critical, Managerial Imaginery, Management, Leadership 


\section{EL VALOR DE LA EFICIENCIA EN LA TRADICIÓN EDUCATIVA}

En el ámbito de la educación formal, ${ }^{1}$ la práctica docente ha estado tradicionalmente regida por una lógica instrumental que otorga un lugar de privilegio a los objetivos educacionales. Son estos los que orientan la selección de los temas que hay que enseñar y organizan la secuencia de actividades en el aula. Proporcionan, además, los parámetros que se adoptan para evaluar los niveles de aprendizaje alcanzados por los estudiantes.

En su análisis comparativo sobre los enfoques de la enseñanza, Fenstermacher y Soltis (1999) enfatizan las analogías entre la educación escolarizada y la actividad empresarial. Señalan que a diferencia del «docente terapeuta» (comprometido con el desarrollo de cada alumno en su propia singularidad) y del «docente emancipador» (que apunta a cultivar una perspectiva crítica libertadora de la mente de los alumnos), el «docente ejecutivo»-perfil predominante en la mayor parte de las escuelas y universidades- centra su atención, al igual que un gerente, en los caminos más eficientes para concretar las metas. Su labor consiste en fijar objetivos precisos, elegir tareas que permitan optimizar el tiempo, establecer parámetros de rendimiento y medir los resultados obtenidos.

Esta suerte de «obsesión por la eficiencia» (Gimeno Sacristán, 1982) ha sido cuestionada en las últimas cuatro décadas desde diferentes disciplinas. En el campo de la Didáctica, Gimeno Sacristán y Pérez Gómez (1992) desnaturalizan la supuesta relación causal entre enseñanza eficaz y aprendizaje. Popkewitz (1994) y Astolfi (2000), por su parte, advierten que a pesar de las intenciones educacionales estrictamente formuladas y de las secuencias de aula minuciosamente diseñadas, la escuela genera en los estudiantes efectos no deseados difíciles de anticipar. En el campo de la Antropología, Lave (1982, 2011) y Lave y Wenger (1991) recuperan el potencial de la vida cotidiana para producir aprendizajes aun en ausencia de un diseño pedagógico riguroso.

Como resultado de estos y otros desarrollos teóricos, la agenda de la Didáctica ha reconsiderado sus preocupaciones centrales (Litwin, 1997) e

1 «Educación formal» alude a la enseñanza impartida en instituciones como escuelas o universidades. La distinción entre educación formal y educación no formal aparece explicitada por primera vez en las actas de la International Conference on World Crisis in Education celebrada en Virgina en 1967. Retomada y profundizada en la obra posterior de Philip Coombs (experto en Educación Comparada y director del International Institute for Education Planning de la unEsco entre 1963 y 1968) operó desde entonces validando y reforzando la distinción - naturalizada en el sentido común- entre instituciones educativas regidas por metas explícitas e instituciones no educativas en las que se aprende de manera espontánea e imitativa. 
introducido nuevas dimensiones en el desempeño del sistema educativo. Sin embargo, la obsesión por la eficiencia se renueva y fortalece año tras año en un ámbito educativo particular: el de la capacitación ejecutiva.

En los ámbitos empresariales -al menos en Argentina- el término «capacitación ejecutiva» alude a un conjunto de programas de formación en temáticas del management ${ }^{2}$ no regulados por la Comisión Nacional de Evaluación y Acreditación Universitaria (CONEAU). Estos programas - a diferencia de los de Maestría o Doctorado- no otorgan certificación académica, de modo que no requieren de la aprobación de exámenes que den cuenta del aprendizaje. Sus destinatarios -en general, directores o gerentes de empresas- no disponen de tiempo para estudiar o profundizar los temas trabajados en el aula. Buscan en estos cursos una actualización en cuestiones relevantes para la función gerencial, así como también un intercambio entre colegas que posibilite enriquecer su experiencia con la de otros. Sin embargo, la definición de la propia inscripción y el costo de la matrícula no suele quedar a cargo de los cursantes. Son generalmente las empresas las que envían a sus gerentes a capacitarse y se hacen cargo del pago de los aranceles. Esto significa que, en la práctica, quien evalúa la oferta de capacitación y elige cuál institución contratar es el director de Recursos Humanos o el funcionario responsable de gestionar el presupuesto corporativo de formación.

En un mercado competitivo como el de la educación ejecutiva en Argentina,${ }^{3}$ los objetivos educacionales operan como factor de atracción. Procurando seducir a clientes familiarizados con la lógica de la eficiencia e interesados en el retorno inmediato de lo que se invierte en enseñanza, las escuelas de negocios se esmeran en formular propósitos cautivantes que induzcan a comprar. Así, enunciados del tipo "proveer en solo equis meses una visión integral del negocio que permita la maximización del valor», "lograr con veinticuatro horas de cursada que la inteligencia emocional actúe como una onda expansiva en la visión y la estrategia de la

2 El término management (en castellano, "gestión») suele emplearse en un sentido amplio y en un sentido estricto. En sentido amplio se lo utiliza para aludir al conjunto de saberes vinculados con el quehacer - la gestión- empresarial. En sentido estricto, designa el conjunto de prácticas de conducción de personas ligado a las teorías clásicas de la administración. En este párrafo uso el término en su sentido más amplio.

3 La oferta de capacitación ejecutiva existe en el país desde 1960. Sin embargo, en la década de 1990 experimentó un crecimiento exponencial como consecuencia del giro neoliberal de la política económica. El ideario del neoliberalismo -que coloca en primer plano la libre competenciafortaleció la creencia en una relación lineal entre capacitarse y desarrollar ventajas competitivas. Surgieron así numerosas instituciones educativas que ofrecían a los individuos el óptimo desarrollo de su «empleabilidad", y a las empresas el cultivo de capacidades indispensables para desempeñarse con éxito en los mercados. 
empresa» o "provocar con un taller de dos días un cambio de comportamiento que conduzca al gerente a ser modelo de su gente» - por citar unos pocos ejemplos $-{ }^{4}$ parecen elaborados para cumplir dos funciones. Especifican el producto final en el que el «cursante-materia prima» supuestamente se transformará por haber participado en el programa, a la vez que estimulan la decisión de compra.

El potencial persuasivo de las metas de enseñanza parece ratificado por los especialistas en marketing educativo. Manes (2004a;2004b) señala que todo brief publicitario que aspire a generar impacto en la venta debe explicitar los objetivos educacionales y sintetizar los puntos clave del servicio ofrecido. Bur (2014) recomienda formular las promesas de enseñanza de una manera tan atrayente que el usuario perciba en ellas una propuesta de valor superadora de la oferta de las otras instituciones. Desde los principales medios gráficos consultados por el público empresario, los expertos advierten que en el «terreno de la educación corporativa - dinámico y competitivo - ganarán los jugadores capaces de mostrar una óptima relación entre precio/prestación/retorno de la inversión» (Clarín Economía, 18 de julio de 2010).

Menos nítida resulta, en cambio, la capacidad de tales enunciados para orientar la tarea docente. ¿De qué manera estructura un profesor la actividad de aula para lograr que la inteligencia emocional actúe como "onda expansiva» en la organización? ¿Qué significa para él semejante enunciado, y qué significa para quienes cursan? Habida cuenta de las características de estos programas -que se desarrollan en horario laboral y no prevén tiempo adicional para tareas académicas-, ¿cómo es posible cumplir con el compromiso de lograr que en escasos meses los cursantes maximicen el valor en las empresas que los integran? ¿En qué consiste el proceso mediante el cual quien asiste a un taller concluye transformado en modelo de su gente?

Preguntas de esta índole solo pueden responderse mediante un abordaje etnográfico ${ }^{5}$ que, más allá de las declaraciones institucionales formales,

4 Las citas están construidas en base a las páginas web de las escuelas de negocios argentinas más prestigiosas, según el ranking 2014 de la revista América Economía.

5 El abordaje etnográfico apunta a comprender las prácticas y significaciones de un grupo particular mediante la inmersión del investigador en la colectividad bajo análisis. A partir de su participación en las actividades del grupo (observando, interactuando), la persona del etnógrafo interviene activamente en la producción del dato: su sola presencia da lugar a que surjan ciertos diálogos o se produzcan ciertas situaciones. Por eso en el género textual conocido como etnografía, el empleo de la primera persona permite al lector reconstruir la evidencia que fundamenta la argumentación del autor (Jacobson, 1991). En este artículo muchas de las expresiones entre comillas se reservan para expresiones textuales de los nativos. 
dé cuenta de la perspectiva de los actores y de sus propios modos de habitar los cursos. En esta línea se inscribe el presente artículo. ${ }^{6} \mathrm{~A}$ partir del registro de campo correspondiente a una clase de Liderazgo, este trabajo aborda la relación entre los objetivos educacionales (el "para qué» de la enseñanza), el contenido (el "qué» de la enseñanza) y las acciones e interacciones dentro del aula. Intenta mostrar la tensión que se establece entre la lógica de la eficiencia -que estructura la tarea del sistema educativo y que actúa como argumento de venta en el entorno de la capacitación ejecutiva- y la lógica vincular de un «imaginario managerial» (Alonso y Fernández Rodríguez, 2006) que tácitamente condiciona la actividad de docente y cursantes.

\section{EL POWER POINT Y LA VIDA REAL: DISTINTAS VOCES DENTRO DEL AULA}

Un martes de 2011 asisto a presenciar la penúltima clase de Liderazgo, uno de los módulos del programa Conducción de Personas que el Instituto Universitario de Formación para Empresas (IUFE) ofrece en su campus situado a $60 \mathrm{~km}$ de Buenos Aires. Salvador, el profesor, es sociólogo especializado en Negociación y magíster en Educación de la University of Bristol. Dirige una importante firma consultora y ejerce la docencia en las escuelas de negocios más prestigiosas de América Latina.

Dos meses atrás, cuando las autoridades del IufE le comentaron que yo realizaría precisamente allí el trabajo de campo correspondiente a mi tesis, ${ }^{7}$ Salvador se mostró interesado en colaborar conmigo. Me invitó a presenciar sus clases y me concedió una extensa entrevista, en la que hablamos sobre sus objetivos educacionales. Hizo alusión entonces a su preocupación por lograr que los cursantes cambiaran la narrativa para pensarse a sí mismos y modificaran en consecuencia su forma de actuar en las organizaciones. Esperaba - dijo- que cada uno advirtiera sus oportunidades de mejora y adquirieran recursos internos para cambiar su propia ac-

6 En línea con las premisas propias del abordaje etnográfico, este artículo no intenta generalizar ni extrapolar los hallazgos a otras clases ni a otras escuelas de negocios. Procura dar cuenta de una práctica social específica y reflexionar sobre lo que el campo revela a la luz una perspectiva teórica particular.

7 Mi tesis de Maestría en Antropología Social (IDES-IDAES/UNSAM) se titula «La gestión del cariño. Una etnografía sobre el aprendizaje y la enseñanza en una escuela de negocios del Gran Buenos Aires». Fue dirigida por Patricia Vargas y Rosana Guber, publicada en 2015 por la editorial Antropofagia de Buenos Aires. A petición de los actores (que solicitaron mantener la confidencialidad) utilizo aquí nombres ficticios. 
tuación en el ámbito de trabajo. Cuando le pregunté por los resultados obtenidos hasta el momento me confesó que se sentía «escéptico»:

En la vida organizacional, la gente encuentra otras realidades, otras prioridades. [...] Salís de acá, volvés a tu empresa, ahí te encontrás con que los demás esperan ciertas cosas de vos, $[\ldots]$ y a la hora de poner en práctica tenés pocos incentivos para el cambio. En el programa ves una oportunidad de mejora, trabajás tus recursos para el cambio, pero en tu organización las condiciones para el cambio no están dadas.

Agregó que, de todas maneras, los participantes regresaban muy satisfechos y recomendaban a sus colegas que se inscribieran en Conducción de Personas: «Ésta, 2011, es ya la cuarta edición del programa. No nos ha disminuido la matriculación».

El grupo de participantes ${ }^{8}$ inscritos en Conducción de Personas está integrado por dieciocho individuos, hombres y mujeres que ocupan posiciones de mandos medios en empresas medianas o en grandes corporaciones. Compartí con ellos cuatro encuentros anteriores, durante los cuales los vi intervenir en actividades áulicas de la más diversa índole: algunos juegos disparadores, ${ }^{9}$ varios role play, ${ }^{10}$ análisis o construcción de casos, ejercicios en subgrupos, trabajos de reflexión personal. A medida que fueron habilitándome el acceso comencé también a intervenir en sus conversaciones informales y a participar de sus desayunos y almuerzos. Hoy, segundo martes de junio, concluye el período de cursada. Nos reencontraremos dentro de tres meses en el Taller de Seguimiento, durante el cual los cursantes conversarán sobre los resultados obtenidos al intentar llevar sus aprendizajes a la práctica.

La cómoda y amplia sala en la que se desarrollan las clases está armada como para facilitar la constitución de subgrupos de trabajo. Ocupan el espacio cinco mesas con capacidad para cinco o seis personas, rodeadas por sillas móviles que permiten modificar la configuración del lugar según lo requieran las distintas tareas.

8 «Participante» suele ser el término que se utiliza para designar a los cursantes. Existe un acuerdo tácito en cuanto a que alumno remite a una situación escolar y asimétrica poco compatible con la paridad y el intercambio de experiencias que los programas de capacitación de adultos apuntan a construir.

9 Es usual que en Capacitación Laboral se utilice el término «disparador» para referirse a actividades de carácter lúdico que operan como "puerta de entrada» (Gardner, 1993) al contenido a aprender. Puestos al servicio del aprendizaje, estos disparadores cumplen dos funciones. Por un lado, permiten a los sujetos que aprenden evocar saberes previos. Por el otro, proporcionan una base vivencial sobre la cual reflexionar luego a la luz del marco conceptual elegido por el docente como tema de enseñanza.

10 El role play (juego de roles) consiste en una actividad durante la cual el aprendiz dramatiza una situación con el propósito de ejercitar y luego analizar una determinada manera de conducirse. 
A las 09:10 de la mañana los dieciocho cursantes están ya sentados en torno a las mesas, esperando la llegada de Salvador. Reina un ambiente cálido, alegre y a la vez algo nostálgico, profuso en comentarios del tipo "qué buena la red que hemos armado» o "tratemos de no perdernos de acá a septiembre». Algunos analizan retrospectivamente su propia cursada y rememoran incidentes del programa. Eleonora comenta cómo fue que resolvió inscribirse: «En el fondo, y esto mi gerente no lo sabe, vine a aprender a venderme y a conocer gente. Para todo se precisan contactos... las redes siempre ayudan». Mauro, en cambio, nos recuerda que Recursos Humanos lo anotó a pesar de sus intentos de resistencia: «De todas maneras al final se me hizo súper llevadero», agrega, «eso se lo debo al grupo, y a que los profesores te la hacen liviana».

Minutos después ingresa al aula el profesor, quien propone a la gente un ejercicio orientado a revisar los contenidos de las clases previas. Les muestra cuatro carteles de cartulina, cada uno de los cuales contiene una leyenda distinta: muy de acuerdo, parcialmente de acuerdo, en desacuerdo y no sabe/no contesta. Distribuye los carteles en los cuatro rincones de la sala y explica:

Yo voy a ir leyendo diferentes enunciados que tienen que ver con temas que vimos acá. Ustedes se van a ubicar en el rincón que mejor refleje su punto de vista. Si están muy de acuerdo con el enunciado, buscan el rincón donde puse el cartel que dice «muy de acuerdo». Si están parcialmente de acuerdo, buscan el rincón del cartel que dice "parcialmente de acuerdo». ¿Se entiende?

Los participantes asienten, y entre todos reacomodan las sillas para poder desplazarse hacia los distintos rincones. Salvador lee entonces el primer enunciado: «La función de los líderes es decir a sus colaboradores qué tienen que hacer».

La gente escucha, se queda unos minutos pensando, y camina entonces al rincón donde está apoyado el cartel que representa su perspectiva. El $80 \%$ se ubica en "parcialmente de acuerdo" y el 20\% en "en desacuerdo». Marcelo, uno de los cursantes, comenta: «La frase es tajante. Para mí, sí. La función de un líder es ejercer el poder». Responde un compañero -ubicado junto al letrero "parcialmente de acuerdo»- que «[...] en parte sí. Cuando nada te funciona y te fallaron todos los intentos que vimos acá, entonces sí. El líder tiene que marcar el campo de juego y ejercer el poder». Interviene el profesor:

Al inicio del programa habíamos hablado de que no hay que confundir poder formal con poder informal, ¿no? Poder informal es compromiso, no jerarquía.Algunos de ustedes 
piensan que no siempre hay que decir qué hacer. Que es preferible acompañar a un grupo, generar las condiciones, en un clima de confianza.

Daniel, también parcialmente de acuerdo con el enunciado, señala:

Vas agotando instancias. Si te ponés de acuerdo con tu colaborador, ahí es puro compromiso y confianza. Si no te ponés de acuerdo, le tenés que decir qué hacer.Ante el desorden, hay que ayudar generando una situación de orden y poner foco.

Salvador insiste: «Tengan en cuenta que en este programa surgió ya varias veces el tema de la visión compartida, de generar condiciones para motivar...». Lo interrumpe Marcelo para insistir a su vez: «Sí, hablar se habló. El tema es que en la realidad a veces no te funciona». El docente intenta advertirle: «Ojo... cuidado que la autoridad formal tienta, ¿no?... La tentación de sentarse en la silla del poder es muy grande...». Propone seguir adelante con el ejercicio y lee la segunda frase: «Toda conversación de feedback con un colaborador sobre aspectos a mejorar debe mencionar también las fortalezas».

El 50\% del grupo se desplaza hacia el cartel «muy de acuerdo». El 30\% se ubica en "parcialmente de acuerdo". Hay un $18 \%$ en desacuerdo y un $2 \%$ de abstenciones. Nuevamente toma la palabra Marcelo: «Sí, yo estoy de acuerdo. Hay que decirle una fortaleza para bajarle la guardia. Cuando ya le bajaste las defensas, después te va a escuchar mejor lo que necesitás que corrija».

Eleonora levanta la mano: «Yo también estoy de acuerdo pero por otra razón. Si no partimos de la fe en las fortalezas del otro, solamente marcamos la falta. No tenemos que caer en ver solamente lo que el otro hace mal. Siempre el otro hace algo bien, y hay que decírselo». Interviene Cati, parcialmente de acuerdo con el enunciado:

A mí no me cierra el sandwichito: «Te acaricio y te doy una buena, te castigo y una mala, otra buena...». Si es una charla puntual en la que tengo que decirle que está trabajando mal, lo que hace bien no viene al caso. Hay conversaciones donde simplemente tengo que decir «hacés todo mal». No es tonta la gente. Si el señalamiento de las fortalezas no es genuino, se dan cuenta.

Luciana está, junto a Marcelo, en el rincón de «muy de acuerdo» y quizás un poco incómoda con el desarrollo de la conversación. Hace saber que quiere contar una experiencia, aunque es consciente de que recibirá críticas: 
Tengo un vendedor muy formado... que no vendía, explica. Buen trato, un profesional, pero no vendía. Lo tuve que agarrar y decirle: si a fin de año no cumplís las metas de ventas, te voy a echar. ¡Demuéstrame que merecés quedarte! Terminó rankeado el mejor vendedor del año. Así que... ¿ ¿cuál es la moraleja? Necesitaba presión. Yo había usado todos los manuales de liderazgo: los que aprendí acá y los que aprendí antes. Pero recién obtuvo resultados cuando lo presioné.

Un compañero bromea: «Sos una bestia, pero con tu gente te sirve». El grupo ríe. Interviene Mauro -quien suele autodenominarse «descreído»para defender a Luciana:

No te sientas mal, porque con la brutalidad lo cuidaste. No tiene que darte culpa. No podemos sacar las cosas de contexto. ¿Cómo se hablan los varones entre sí en una metalúrgica dura? ¿Cómo se tratan? Así como estamos viendo en las clases, no: te tomarían por maricón. Así que depende.Todo depende. Una cosa es los libros, o el Power Point; otra distinta es la vida real en las empresas.

Se generan varias conversaciones en simultáneo y el profesor sugiere pasar al tercer enunciado: «En una reunión, los líderes por sobre todas las cosas transmiten sus ideas con firmeza y seguridad, logrando una escucha atenta e interesada de las otras personas». Los participantes se distribuyen de manera más o menos uniforme entre los cuatro rincones. Uno de los que se declara en desacuerdo expone que la función predominante de un líder consiste en escuchar, no ya en transmitir. Lo contradice un compañero arguyendo que el líder tiene que ser capaz de tomar decisiones firmes que marquen el rumbo de cada proyecto. Toma la palabra Salvador:

Vean esto. Hay gente que se está expresando y los demás están logrando escuchar atentamente al grupo. Nadie duda de que sea una habilidad, esto de escuchar. Escuchar distintas voces... Esto es muy valioso porque todos tenemos distintas voces, pero para trabajar juntos tenemos que llegar a acuerdos. Decimos, pensamos, valoramos cosas distintas... incluso vemos cosas distintas. ¿Cómo conseguimos un acuerdo cuando lo que yo veo es una cosa y la otra persona ve otra?

Un muchacho responde que es necesario indagar para entender qué ve el interlocutor, y argumentar claramente para explicar qué vemos. El profesor asiente, y reflexiona: «Es cierto, pero tengamos en cuenta que para lograr acuerdos no basta con que cada uno entienda al otro. Insisto en esto: ¿cómo logramos acuerdos si estamos viendo cosas distintas?».

Contesta Marcelo destacando que, cuando las dos personas ocupan posiciones similares en el organigrama, se hace más fácil. En caso contrario, «el que está arriba» seguramente trate de convencer a quien está «abajo» porque esa es la manera en la que funcionan las empresas. 
A veces querés que tus colaboradores acepten las cosas no por obligación sino por convicción. Bueno; no podés. Así te lo digo. No podés, enfatiza. La racionalidad de la organización es otra [...]. Los accionistas quieren que la compañía gane plata. Si el empleado está de acuerdo, excelente. Si no, que se vaya... o se quede haciendo lo que hay que hacer. ¿A largo plazo se daña el vínculo? Y bueno, que se dañe. Total, la subsistencia de la compañía depende del corto, y la cabeza de los gerentes va a depender del corto. Entonces como gerente podés probar con todas las recomendaciones de todos los manuales de Coaching pero todo tiene un límite.

Salvador pide opinión a los demás. Eleonora sostiene que a pesar de su confianza en el Coaching y en los contenidos del programa, tiene que reconocer que su compañero tiene «bastante razón». El profesor contesta que el argumento de Marcelo es válido, aunque reitera su interés en que el grupo aprenda a «manejar una conversación difícil, escuchando y concediendo al colaborador y al mismo tiempo cuidando el mensaje que hay que decir porque este es el desafío del liderazgo: no quedarnos atrapados en el falso dilema». Toma entonces tres sillas, las ubica en hilera de izquierda a derecha y prosigue con su explicación:

Una alternativa del falso dilema es sentarme en la silla de la agresividad [dicho lo cual se sienta en la primera de las sillas]. El tema acá es que la otra persona puede sentirse criticada, cuestionada. El extremo contrario [se sienta en la tercera de las sillas] es decir las cosas con tal vaguedad que el otro no entienda... endulzar el mensaje. Con semejante vaguedad el otro no recibe mi influencia. La otra silla que supera el falso dilema es la silla de la comunicación. [Se sienta en la silla del medio de las tres]. O sea: te hablo con pausas, tranquilo, pero firme y claro. El otro me ve claro, puede entenderme, pero no se siente presionado. Ahí ya estamos en condiciones de comenzar a construir un acuerdo del tipo «vos y yo».

Lo interrumpe Mauro: «Perfecto. El otro te ve claro, pero igual se resiste. Entonces ahí la única que te queda es poner un límite: flaco, acá las cosas son así, y si no te gusta ahí está la puerta». El grupo explota en risas y exclamaciones. Retoma entonces Mauro en un tono menos exaltado:

Perdón, pero si tengo que ser franco yo solamente aplico el $30 \%$ o menos de las cosas que se ven en estos cursos. A veces pienso que tendría que agarrar la carpeta y volver a leer, pero la verdad... ¿para qué? Las empresas son lo que son y yo mañana tengo que ir a trabajar.

Eleonora señala que si bien es cierto que la realidad no es igual a los libros:

Tenés la ilusión de que alguna idea pueda ayudar a cambiar un poquito y algo se mueva en tu cabecita. Es cuestión de seguir leyendo, aprendiendo. Por eso yo espero que antes 
del taller de septiembre sigamos en contacto aunque sea por teléfono o mail.Así, siempre alguno te puede ayudar a encender una nueva llamita.

Concluye el docente, indicando que el programa no apunta a cambiar la realidad sino a «hacerte cargo de lo que te pasa a vos y a desarrollar tus propios recursos». Indica que en un rato les propondrá un ejercicio orientado a que puedan diseñar un plan de mejora personal, integrando los principales aprendizajes del programa: «Voy a invitarlos a ver qué querrían llevar a la práctica de acá a septiembre. Qué actitudes o comportamientos querrían cambiar o incrementar, en qué situaciones concretas. Pero antes...»-advierte - «... antes hagamos el coffee-break, que hemos trabajado muy intensamente en esta primera parte de la mañana».

\section{EL CONTENIDO DE LA ENSEÑANZA Y EL «IMAGINARIO MANAGERIAL»}

El precedente registro de campo da cuenta de dos maneras diferentes de entender el trabajo de conducir equipos: el que plantea Salvador, y el que - al menos de a ratos- enuncian los cursantes. Según el temario de la clase, el desafío del liderazgo consiste en lograr compromiso prescindiendo del poder formal que procede de la jerarquía. Para esto es preciso que el líder ponga en juego un conjunto de habilidades para el logro de acuerdos tales como escuchar, indagar, y establecer relaciones de paridad prescindiendo de la silla de la autoridad formal. Gracias a estas habilidades logrará acuerdos superadores de los conflictos sobre los cuales edificar la armonía del grupo.

Este modo de concebir a la conducción resulta consistente con el modelo de liderazgo que propone la literatura sobre empresas de los últimos treinta años. Sus autores - procedentes del mundo angloparlante o fuertemente influidos por el pensamiento estadounidense sobre management $-{ }^{11}$ coinciden en recalcar que, en entornos como los actuales (calificados en general de turbulentos o altamente competitivos), se requiere de una modalidad de gestión diametralmente opuesta a la que estaba vigente durante la era industrial de la primera mitad del siglo xx.

11 La influencia de estas corrientes de pensamiento en América Latina y particularmente en Argentina -a la que no voy a referirme en este trabajo- está claramente expuesta en los trabajos de Szlechter (2013), Gantman y Fernández Rodríguez (2008) y Luci (2011). 
Consideremos, a modo de ejemplo, uno de los planteamientos más difundidos en Argentina: el del especialista chileno Rafael Echeverría. ${ }^{12} \mathrm{El}$ autor traza una nítida comparación entre la «empresa tradicional» y la «empresa emergente»:

\begin{tabular}{lll}
\hline Criterios & $\begin{array}{l}\text { Empresa } \\
\text { tradicional }\end{array}$ & $\begin{array}{l}\text { Empresa } \\
\text { emergente }\end{array}$ \\
\hline $\begin{array}{l}\text { Trabajo preponderante } \\
\text { Fundamento del trabajo }\end{array}$ & $\begin{array}{l}\text { Trabajo manual } \\
\text { Destreza física }\end{array}$ & $\begin{array}{l}\text { Trabajo no manual } \\
\text { La palabra }\end{array}$ \\
$\begin{array}{l}\text { Clave de la productividad } \\
\text { ción }\end{array}$ & Movimientos y tiempos & $\begin{array}{l}\text { Competencias conversa- } \\
\text { cionales }\end{array}$ \\
Locus reflexivo & Línea de ensamblaje & Rediseño de procesos \\
Mecanismo de regulación & Mando y control & Autonomía responsable \\
Carácter de la regulación & Techo & Piso \\
Emocionalidad de base & Miedo & Confianzo: el practicante \\
Perfil de autoridad & Capataz & Coach \\
Tipo de organización & Piramidal y jerárquica & Horizontal y flexible \\
Criterio guía & Estandarización & Aprendizaje organizacional \\
\hline
\end{tabular}

(Echeverría, 2000: 101)

Sostiene que la mayor parte de los problemas de desempeño empresarial radica en la discrepancia entre los requerimientos de la "empresa emergente» y los paradigmas de gestión aprendidos:

12 Rafael Echeverría es profesor universitario y socio fundador de la consultora Newfield Consulting, que opera en Estados Unidos, España,Argentina, México, Brasil,Venezuela, Colombia y Chile. Entre sus principales publicaciones se encuentran Ontología del lenguaje (1994) y La empresa emergente (2000).Ambos textos forman parte de la bibliografía citada por los docentes del programa Conducción de Personas del iufe. 
estamos atrapados en un modelo de gestión que sigue remitiendo a lo que [Frederick] Taylor ofreció al problema de la productividad del trabajo manual [...]. Es el modelo de gestión lo que está trabando una capacidad de desempeño... que creemos que hay que liberar... abrir. Esto implica el tránsito de lo que nosotros hemos llamado «la figura del gerente capataz» a lo que se ha llamado en el debate empresarial de los Estados Unidos "gerente coach». Un gerente que descansa en su capacidad de escucha, de indagación, de diseñar conversaciones, de intervenir en los espacios emocionales de sus equipos de trabajo (Echeverría, 2013).

Por consiguiente, todo líder que aspire a mejorar el rendimiento del equipo a su cargo tiene que abandonar las habilidades propias del «capataz» y cultivar el ejercicio del coaching.

En una línea afín, el argentino Fred Kofman ${ }^{13}$ coincide con Echeverría en distinguir entre un antes y un ahora en la práctica de la conducción de equipos. Señala que tradicionalmente - desde los antiguos mitos heroicos hasta la literatura empresarial con la que estamos familiarizados- el líder fue identificado con el ejercicio de la autoridad formal. Sin embargo, «en situaciones altamente inciertas, el liderazgo compartido tiene ventajas superiores a las del liderazgo individual» (Kofman, 2010). Compartir el liderazgo significa, para el autor, lograr que el equipo a cargo opere como:

más que la suma de sus integrantes. Lo que diferencia a un equipo de un grupo de gente que trabaja es la sinergia.A través del desarrollo de una visión compartida, de un compromiso con ciertos valores esenciales, un contexto de confianza y respeto mutuos [...], un grupo de individuos puede generar una energía creativa muy superior a la mera adición de energías individuales.Así como un haz de luz puede mediante un cristal organizarse en un rayo láser, un haz de individuos puede -a través de un campo de fuerzas intelectuales, emocionales y existenciales- producir un equipo extraordinario. El líder es la persona a cargo de crear y mantener tal campo de fuerzas (Kofman, 2010).

Los cursantes, sin embargo -al menos ocasionalmente durante la clasehacen referencia a una realidad distinta. Su experiencia cotidiana les indica que, más allá de los libros o el Power Point, la actividad empresarial se sustenta sobre una lógica económica ("que la compañía gane plata») y una jerarquía que enmarca la interrelación entre el de arriba y el de abajo. Las preferencias de los trabajadores no siempre coinciden con los cursos de acción más rentables, y «la cabeza del gerente» depende de su capacidad para lograr que el empleado se vaya... o se quede haciendo lo que hay que

13 Fred Kofman se graduó en Economía en la Universidad de Buenos Aires y obtuvo un Doctorado en la misma disciplina en la University of California, Berkeley. Durante varios años se dedicó a la consultoría y a la docencia en diversas escuelas de negocios (el MIT, entre otras). Actualmente ejerce como vicepresidente de Desarrollo Ejecutivo en LinkedIn. Entre sus textos más difundidos se encuentra Metamanagent (2011) y La empresa consciente (2012). 
hacer. Por lo tanto, los intentos del líder por lograr una armonía duradera -inviable, habida cuenta de la contraposición de intereses- quedan reducidos a artilugios orientados a que el equipo baje la guardia y acepte las reglas de juego.

Ahora bien: si los comentarios de los cursantes remiten a las empresas concretas en las que se desempeñan día a día, ¿a qué organizaciones alude la bibliografía sobre management? ¿Cuál es y dónde se encuentra esa organización en la que todos comparten el liderazgo y confluyen en un campo de fuerzas intelectuales, emocionales y existenciales? ${ }^{14}$

Desde una perspectiva crítica -y otros marcos disciplinares-, trabajos como los de Boltanski y Chiapello (2002), Alonso y Fernández Rodríguez (2006) y Gantman (2009) coinciden en la respuesta a estas preguntas. Los autores sostienen que la literatura empresarial no remite a organizaciones concretas porque no describe ni explica fenómeno alguno; más bien se encarga de enunciar un tipo de realidad que aspira a crear. Se trata, por consiguiente, de un «conjunto textual con vocación preformativa» (Boltanski y Chiapello, 2002: 101) que naturaliza una perspectiva particular y prescribe determinadas maneras de actuar y pensar. Esto significa que la pregunta por la realidad fáctica de la empresa emergente debe ser reemplazada por otra, relativa a los propósitos que guían la enunciación de una organización de tales características.

Alonso y Fernández Rodríguez (2006) advierten sobre la dimensión imaginaria en el desarrollo del pensamiento administrativo. A través de un análisis sobre lo que ha significado "gestionar»en las diferentes etapas del capitalismo, identifican dos configuraciones de un «imaginario managerial» que condensa las preferencias y valores propios de cada época histórica. La primera de estas configuraciones está asociada al industrialismo del siglo xx, a la producción en serie y a la intervención del Estado en la esfera económica. La segunda se vincula con la fragmentación de la demanda y la producción a medida que introdujo Japón desde comienzos de los ochenta. Ambas cuentan con sus propios recursos semánticos para conseguir la adhesión de quienes gestionan. En la configuración asociada al industrialismo se destaca la noción de "progreso», entendido como conjunto de beneficios colectivos a los que la producción organizada según las reglas de la Administración Científica supuestamente conduciría. En aquella asociada a la producción a medida cobra énfasis la idea de personalización encarnada

14 Varias publicaciones ejemplifican sus planteos apelando a historias de éxito. Sin embargo, la selección del caso y su narración reflejan exclusivamente la perspectiva del autor: ni los empleados ni los mandos medios de tales empresas supuestamente exitosas intervienen en la escritura de la historia. 
en la figura de un colaborador que se implica de manera personal y voluntaria con la empresa en la que trabaja.Así, estas construcciones discursivas han articulado los valores y las convenciones sociales de cada época con los requerimientos de la rentabilidad mercantil.

Cuando el capitalismo de emprendedores generó problemas al no poder abastecer a los mercados, nacieron los sistemas de producción en serie [...] El imaginario está repleto de jerarquías, instrumentos de control, celdas, barreras entre tareas, planos, esquemas, etc. [...] Cuando el modelo de organización taylorista y fordista entró en crisis, con una evidente caída de la productividad, [...] el management del cambio de los 80 propuso una transformación radical, basada en fomentar la participación [...] de todas las personas de la organización, anulando las viejas lealtades colectivas (sindicales) y estatales (Alonso y Fernández Rodríguez, 2006: 145).

A la luz de este análisis, la discrepancia entre Salvador y los cursantes cobra otro sentido. En tanto comparten un «imaginario managerial» de época y confluyen en una escuela de negocios, docente y participantes podrán -al menos durante un rato y dentro de las cuatro paredes del aulahacer como si las asimetrías y los conflictos hubiesen sido reemplazados por equipos sinérgicos con visiones compartidas. Sin embargo, toda vez que la realidad irrumpe pone de manifiesto que las organizaciones horizontales y armónicas existen solamente en los libros o el Power Point. ${ }^{15}$

\section{OBJETIVOS EXPLÍCITOS Y PRESCRIPCIONES TÁCITAS}

La página web del Iufe enuncia de manera explícita los objetivos educacionales del programa Conducción de personas. Indica que, al finalizar la cursada, los destinatarios habrán podido: «desarrollar una visión más amplia de sí mismos como líderes, movilizar a personas diversas hacia los requerimientos del negocio y [...] preparar a los equipos del mañana». Salvador, por su parte, espera que el módulo a su cargo (Liderazgo) permita a los cursantes cambiar la narrativa sobre sí mismos para poder en consecuencia actuar de manera diferente. Para que todos estos propósitos resul-

15 No cabe duda de que las modalidades de producción han cambiado y que las organizaciones posfordistas difieren de la línea de montaje no solo en la dimensión imaginaria sino también en las prácticas y sistemas de organización del trabajo. Esto no significa que las descripciones ofrecidas por la literatura empresarial -que centran el foco en la ausencia de conflicto en la horizontalidad, en la capacidad de las empresas para promover la motivación o en el cuidado de salud emocional de los trabajadores - den acabada cuenta de las características reales de las organizaciones concretas. 
ten factibles es preciso que el trabajo en el aula se estructure en torno a dos focos: la revisión conceptual y la comprensión del contenido.

El primero de ellos - la revisión conceptual- se vincula con lo que en el campo de la Didáctica se conoce como «cambio conceptual» (Pozo, 2006), «cambio en el modelo mental» (Perkins, 2010) o «rectificación de estereotipos y simplificaciones» (Gardner, 1993). En efecto, para que los participantes puedan entender el planteo del docente se requiere que, ante todo, estén dispuestos a revisar sus creencias y acepten -al menos en algún grado- las premisas básicas del «imaginario managerial». Semejante reconstrucción difícilmente se produzca de manera espontánea, máxime cuando la experiencia cotidiana parece confirmar a los cursantes que los intereses que movilizan a las personas diversas y los requerimientos del negocio no tienen por qué confluir. Haría falta, por lo tanto, un diseño didáctico que colocara en primer plano el «conflicto sociocognitivo» (Astolfi, 2000) entre dos concepciones sobre el rol de quien conduce equipos. Esto implica para el docente la implementación de tareas orientadas a profundizar las contradicciones y a contrastar la epistemología disciplinar del management contemporáneo con la práctica concreta de quienes gerencian.

El segundo de los focos - la comprensión del contenido- remite a un proceso dirigido a lograr un «uso activo» (Perkins, 1995) del conocimiento. No basta con que los participantes se familiaricen con los temas del programa, ni siquiera que los recuerden. Es necesario que se los adueñen de tal manera que el nuevo saber dispare la búsqueda reflexiva de renovados cursos de acción. Para que esto resulte posible, los aprendices tienen que llevar a cabo actividades intelectualmente desafiantes como la comparación, la argumentación, la formulación de preguntas, el descubrimiento de problemas y la reflexión crítica, anticipando la transferencia ${ }^{16}$ y contando con una devolución apropiada que los ayude a monitorear la calidad del razonamiento propio (Perkins y Blythe, 1994).

Sin embargo, la clase de Salvador se orienta en una dirección diferente. Lejos de profundizar el conflicto el profesor resalta la importancia de los «acuerdos del tipo vos y yo»: las discrepancias simplemente se enuncian, sin que las diferentes voces tengan la ocasión de debatir en profundidad. El tiempo en aula resulta al grupo «súper llevadero», aunque sus integrantes

16 En la visión cognitiva del aprendizaje se entiende por transferencia al fenómeno por el cual algo aprendido de un contexto es trasladado a otro contexto (Perkins y Salomon, 1988 y 1992). Gran parte de la investigación en Didáctica atañe precisamente a las estrategias docentes más aptas para promover la transferencia de lo que se enseña en aula a los diferentes entornos de la vida fuera del aula. 
se retiran -luego de dos meses de cursada- tan convencidos como llegaron acerca de la imposibilidad de conciliar «los libros» con la vida cotidiana en la empresa. Como consecuencia, no hay indicios que permitan advertir la contribución del módulo Liderazgo a las promesas expresadas en la página web del Iufe. Cualquier evaluación rigurosa de eficiencia que apuntara a contrastar objetivos y logros pondría en evidencia que, al menos en lo atinente al desarrollo de los contenidos, el resultado puede ser caracterizado en los términos del "conocimiento frágil» (Perkins, 1995). ${ }^{17}$

El docente lo sabe, él mismo se declara «escéptico» al analizar los cambios que desearía promover. Es también consciente de que el principal obstáculo radica en la brecha entre lo que propone su módulo (descubrir oportunidades de mejora y generar recursos para el cambio) y las otras realidades, otras prioridades que los gerentes encuentran en las organizaciones. Sin embargo, el diseño de sus clases -intensas, atractivas para el grupo, ricas en actividades variadas y entretenidas- ofrece pocas instancias de exploración de las tensiones. ${ }^{18}$

Cabe entonces la pregunta acerca de los criterios que orientan la secuencia áulica de Salvador. Si sus propósitos están claros, ¿por qué no operan como guía en la selección de actividades? ¿Qué es lo que explica que, precisamente en un entorno que valora la eficiencia y el control, la relación entre metas y medios resulte tan remota? Sostengo aquí que el «imaginario managerial» ejerce en la tarea docente una influencia muy superior a la que ejercen las intenciones declaradas. Dicho de otro modo: las corrientes actuales del management no solo prescriben el "qué» de la enseñanza sino también el "cómo», relativizando el privilegio que la educación formal tradicionalmente otorgó a los objetivos educacionales.

Recordemos que, según los libros y el Power Point, la principal labor del líder» consiste en acompañar a un grupo sin discutir ni persuadir. La exposición de ideas con firmeza y seguridad puede conducir a que el interlocu-

17 En La escuela inteligente Perkins (1995) caracteriza al conocimiento frágil como poco resistente al olvido, inerte (no puede ser utilizado fuera del aula), ingenuo (no rectifica comprensiones erróneas o estereotipos) y ritual (solo sirve para resolver ejercicios de formato pedagógico en clase). Es posible, sin embargo, que algunos cursantes hayan tomado a su cargo el propio aprendizaje y hayan obtenido (mediante la reflexión, la lectura, el pedido de ayuda a los compañeros de trabajo o actividades variadas fuera del aula) resultados más contundentes.

18 La clase muestra pocos indicios de logro en términos de comprensión y transferencia del contenido. Esto no implica, sin embargo, que no produzca resultados significativos. Tal como los mismos cursantes lo señalan, el docente promueve el aprendizaje entre pares a través del intercambio, facilita la exploración de puntos de vista, y habilita permisos para que cada individuo pueda poner palabras a su propia perspectiva. Por otra parte, esas mismas actividades (role play, juegos disparadores, etc.) resultarían aptas para promover el cambio en los modelos mentales si estuvieran acompañadas por otras que permitieran explicitar y profundizar el conflicto cognitivo. 
tor se sienta criticado y cuestionado. Es necesario entonces evitar la tentación de la autoridad formal y cultivar habilidades dirigidas a empatizar, con miras al acuerdo que todo equipo necesita. Esta argumentación que Salvador enuncia es la misma que actúa cuando, en calidad de líder a cargo del módulo, se aboca a escuchar distintas voces sin confrontar ni convencer. Son los participantes - algunos de ellos visiblemente molestos- quienes introducen el conflicto, ante el cual el profesor responde con las mismas recomendaciones que brinda al grupo: hablar de manera pausada y tranquila, ejercer influencia de un modo sutil y buscar puntos de concordancia.

El módulo de Liderazgo nos revela entonces una lógica alternativa al instrumentalismo del "paradigma proceso-producto» (Gimeno Sacristán y Pérez Gómez, 1992). El profesor enseña, más por poner en acción el «imaginario managerial» que por traducir objetivos en técnicas. Sus dispositivos didácticos hablan por sí mismos con elocuencia, comunicando que un buen líder conduce a su grupo relegando las confrontaciones y colocando en primer plano las actividades orientadas a compartir.

\section{DOS ENCUADRES PARA DOS CLIENTES}

El registro de campo de Liderazgo y las reflexiones posteriores han puesto de manifiesto la tensión entre dos encuadres. Uno de ellos recrea las premisas básicas de una tradición educativa centrada en la eficiencia. Otorga a las metas de enseñanza un lugar de privilegio, y asigna al docente un papel gerencial cuya responsabilidad central radica en el óptimo empleo del tiempo (Fenstermacher y Soltis, 1999). Por su concordancia con la linealidad fabril, este enfoque -cuestionado desde diversas corrientes didácticas- mantiene y renueva su vigencia en el ámbito de la capacitación ejecutiva. Más aún: ante el incremento de la competencia y merced a las recomendaciones del Marketing, las metas educacionales de los programas de formación han adoptado en los últimos años la forma de sofisticadas declaraciones que prometen rápidas y radicales transformaciones en quienes cursen.

Tales enunciados resultan especialmente atractivos para quienes gestionan los presupuestos corporativos de Capacitación. Conscientes de que deben lograr resultados tangibles con un costo acotado y en un tiempo mínimo, estos funcionarios dedican horas y esfuerzo a evaluar comparativamente las distintas propuestas institucionales. 
Los cursantes, sin embargo, juzgan con parámetros diferentes. No suelen ser ellos quienes deciden qué programa de capacitación tomar, cuándo o por qué. La asistencia a cursos elegidos por la empresa constituye un requerimiento laboral como cualquier otro, más o menos interesante según el caso. Puede que algunas personas estén deseosas de recibir formación y dispuestas a embarcarse en tareas exigentes que les permitan aprender. Otros, en cambio, procurarán ser eximidos de la obligación de asistir o tolerarán la cursada en tanto la encuentren llevadera y los ayude a conocer gente.

Aquí cobra entonces protagonismo un segundo encuadre, ensalzado en la literatura actual del management. Contradice la lógica eficientista de los objetivos educacionales y entroniza en cambio una lógica vincular según la cual la confianza, las emociones, los acuerdos armónicos y las redes constituyen la clave para el aprendizaje y para el trabajo en empresas. En este enfoque no son las metas las que guían la selección de actividades de clase. Los role play, los juegos disparadores y los ejercicios grupales no son traídos al aula por su aptitud para promover el cambio conceptual o la comprensión del contenido. Más bien operan como factor de atracción para quienes asistir a una escuela de negocios significa ante todo establecer contactos y construir una trama interpersonal que beneficie a quienes la integran. ${ }^{19}$

El docente no puede optar entre un enfoque o el otro. Prescindir de la racionalidad instrumental equivaldría a perder capacidad de venta, ya que el cliente que compra evalúa cuestiones ligadas a la relación entre objetivos declarados, resultados y costos. Al mismo tiempo, la lógica vincular es lo que permite que el otro cliente - el cliente que cursa- se retire lo suficientemente satisfecho como para recomendar entre sus allegados el programa al que asistió o pedir a Recursos Humanos su inscripción en otros programas. El diseño didáctico tiene que conformar a todos; de ello depende la continuidad del programa y, en última instancia, la de la escuela de negocios que lo ofrece. Al agente educativo le caben entonces las pautas que la literatura empresarial actual prescribe para todo prestador de servicios: producción a medida y atención personalizada a demandas múltiples.

El discurso del management contemporáneo parece actuar como punto de confluencia entre los dos encuadres. En su formato explícito - como contenido curricular- constituye el «qué» de la enseñanza. Confiere funda-

19 Constituyen a la vez dispositivos idóneos para el intercambio entre pares, la exploración de puntos de vista y la reflexión sobre la propia experiencia. Generan así aprendizajes personales e interpersonales más allá de los contenidos disciplinares a los que se apliquen y a las metas educacionales declaradas. 
mento a los objetivos educacionales a la vez que proporciona los recursos retóricos necesarios para un buen marketing. En su formato tácito - como marco normativo que prescribe maneras de actuar y de vincularse- guía la selección de estrategias didácticas otorgando prioridad a aquellas orientadas a conocer gente. De este modo el «imaginario managerial» articula los intereses de actores sociales diversos y posibilita que, año a año, la escuela de negocios mantenga su atractivo en su doble condición de institución educativa y afiliativa.

\section{BIBLIOGRAFÍA}

Alonso, L. y Fernández Rodríguez, C. J. (2006): «El imaginario managerial: el discurso de la fluidez en la sociedad económica», Política y Sociedad, 23(2), pp. 127-151.

Astolfi, J. P. (2000): Aprender en la escuela, Santiago de Chile, Dolmen.

Astolfi, J. P. y Peterfalvi, B. (1997): «Estrategias para trabajar obstáculos: dispositivos y resortes», en CAmilloni, A. (comp.) (1997): Los obstáculos epistemológicos en la enseñanza, Barcelona, Gedisa, pp. 191-223.

Boltanski, L. y Chiapello, E. (2002): El nuevo espíritu del capitalismo, Madrid, Akal.

Bur, A. (2014): «Marketing educativo. Cómo captar, retener y fidelizar alumnos», Reflexión Académica en Diseño y Comunicación, XV(22), pp. 47-50.

Coombs, P. H. (1966): «The International Institute for Educational Planning», International Review of Education, 2(3), pp. 333-345.

- (1976): «Nonformal Education: Myths, Realities and Opportunities», Comparative Educational Review, 20(3), pp. 281-293.

- (1985): La crisis mundial de la educación: perspectivas actuales, Madrid, Santillana.

ECheverría, R. (2002): La empresa emergente, Buenos Aires, Granica.

- (2013): Conferencia de Rafael Echeverría «Lenguaje, emprendimiento y liderazgo», Universidad de Deusto, disponible en: https://www.youtube. com/watch? $=2 \mathrm{D} 560 \times 8 \mathrm{pVQE}$ [Consultado el 17 de noviembre de 2016]

Fenstermacher, G. y Soltis, J. (1999): Enfoques de la enseñanza, Buenos Aires, Amorrortu. 
Gantman, E. y Fernández Rodríguez, C. J. (2008): «Notas sobre la evaluación del conocimiento administrativo en la República Argentina y su comparación con el caso español (1913-2007)", Cadernos EBAPE.BR, 6(4), pp. 115-135.

GaNTMAN, E. (2009): «Reflexiones sobre la evolución de las ideologías gerenciales desde el siglo xIx hasta el presente», Energeia, 5(1), pp. 96-115.

GaRDNER, H. (1993): La mente no escolarizada. Cómo piensan los niños y cómo deberían enseñar las escuelas, Buenos Aires, Paidós.

Gimeno SACRISTÁn, J. (1982): Pedagogía por objetivos: una obsesión por la eficiencia, Madrid, Morata.

Gimeno Sacristán, J. y Pérez Gómez,A. (1992): Comprender y transformar la enseñanza, Madrid, Morata.

Giroux, H. y McLaren, P. (1994): Between Borders. Pedagogy and the politics of cultural studies, Nueva York, Routledge.

Gore, E. y DunlaP, D. (2006): Aprendizaje y organización: una lectura educativa de las teorías de la organización, Buenos Aires, Granica.

JACoBson, D. (1991): Reading Ethnography, Nueva York, University of New York Press.

Kofman, F. (2007): Metamanagement. La nueva conciencia de los negocios, Buenos Aires, Granica.

- (2010): «Shared Leadership. Managing Complexity». Disponible en: http://www.axialent.com/uploads/paper/archivo/Shared_Leadership,_ Managing_Complexity_by_Fred_Kofman.PDF [Consultado el 17 de noviembre de 2016]

- (2012): La empresa consciente. Cómo construir valor a través de valores, Buenos Aires, Aguilar.

KotTer, J. (2005): «Lo que de verdad hacen los líderes», Los clásicos de Harvard Business Review 1990, Harvard Business School Publishing Corporation, pp. 17-25. Disponible en http://educatic2011.files.wordpress. com/2010/10/kotter-2005.PDF [Consultado el 16 de noviembre de 2016]

LAVE, J. (1982): «A comparative approach to Educational Forms and Learning Process", Anthropology \& Educational Quarterly, 13(2), pp. 181-187.

- (2011): Apprenticeship in Critical Ethnographic Practice, Chicago, University of Chicago Press.

LAVE, J. y Wenger, E. (1991): Situated Learning. Legitimate peripheral participation, Cambridge, Cambridge University Press.

LiTWIN, E. (1997): Las configuraciones didácticas. Una nueva agenda para la enseñanza superior, Buenos Aires, Paidós. 
LuCI, F. (2011): «La carrera directiva en el marco de la reconfiguración empresarial argentina: ¿Una revolución managerial?», Revista Latino-Americana de Estudos do Trabalbo, 16(26), pp. 145-181.

- (2011): «Managers de grandes empresas: ¿Trabajadores o patrones?», Apuntes de investigación de CECYP, Centro de Estudios en Cultura y Política, Fundación del Sur, 20, pp. 193-202.

Manes, J. M. (2004a): Ética y marketing de la institución educativa: una conciliación. Disponible en: http://www.orientacionvocacional.com/ index.php/blogs/item/389-etica-y-marketing-de-la-institucion-educativauna-conciliacion [Consultado el 16 de noviembre de 2016]

- (2004b): Marketing para instituciones educativas. Guía para planificar la capacitación y retención de alumnos, Buenos Aires, Granica.

PERKIns, D. (1995): La escuela inteligente, Barcelona, Gedisa.

- (2010): El aprendizaje pleno. Principios de la enseñanza para transformar la educación, Buenos Aires, Paidós.

Perkins, D. y SAlomon, G. (1988): «Teaching for Transfer», Educational Leadership, 46(1), pp. 22-32.

- (1992): "The Science and Art of Transfer». Disponible en http://learnweb.harvard.edu/ALPS/Thinking/docs/trancost.PDF [Consultado el 17 de noviembre de 2016]

Perkins, D. y Blythe, T. (1994): «Putting Understanding up-front», Educational Leadership, 51(5), pp. 4-7.

Popkewitz, T. (1994): Sociología política de las reformas educativas, Madrid, Morata.

Pozo, J. I. (2006): Teorías cognitivas del aprendizaje, Madrid, Morata.

SzlECHTER, D. (2013): «La emergencia de la comunidad gerencial en la Argentina. Una mirada comparativa», Revista del Centro de Estudios de Sociología del Trabajo, 5, pp. 3-46.

- (2013): "La segmentación del mercado interno de trabajo gerencial», Revista Latino-Americana de Estudos do Trabalbo, 18(29), pp. 13-51.

Thrift, N. (2000): «Performing Cultures in the New Economy», Annals of the Association of American Geographers, Taylor \& Francis y Association of American Geographers, 90(4), pp. 674-692.

- (2001): "It's the romance, not the finance, that makes the business worth pursuing: disclosing a new market culture», Economy and Society, 30(4), pp. 412-432.

VÁzquez Mazzini, M. (2015): «Palabras lindas, crudas realidades. Reflexiones sobre la enseñanza y el aprendizaje de la ideología empresarial», Prácticas de oficio. Investigación y reflexión en Ciencias Sociales, 
n. ${ }^{\circ}$ 14. Disponible en http://ides.org.ar/wp-content/uploads/2015/02/ Vazquez-texto.pDF [Consultado el 17 de noviembre de 2016]

- (2015): La gestión del cariño. Una etnografía sobre el aprendizaje y la enseñanza en una escuela de negocios del Gran Buenos Aires, Buenos Aires, Antropofagia. 